\title{
Double Edge-Vertex Domination on Middle and Splitting Graphs of Path and Cycle
}

\author{
Banu Aylı ${ }^{1 *}$ and Elgin Kılıç ${ }^{1}$ \\ ${ }^{1}$ Department of Mathematics, Faculty of Science, Ege University, İmir, Turkey \\ ${ }^{*}$ Corresponding author
}

\section{Article Info}

Keywords: Double edge-vertex dominating set, Double vertex-edge dominating set, Edge-vertex dominating set, Vertex-edge dominating set 2010 AMS: 05C69, 68R10

Received: 15 April 2021

Accepted: 1 October 2021

Available online: 1 December 2021

\begin{abstract}
An edge $e=u v$ of graph $G=(V, E)$ is said to be edge-vertex dominate vertices $u$ and $v$, as well as all vertices adjacent to $u$ and $v$. A set $S \subseteq E$ is a double edge-vertex dominating set if every vertex of $V$ is edge-vertex dominated by at least two edges of $S$. The minimum cardinality of a double edge-vertex dominating set of $G$ is the double edge-vertex domination number and is denoted by $\gamma_{d e v}(G)$. In this paper, we present results for middle graphs of path and cycle and some splitting graphs of path and cycle on double edge-vertex domination numbers.
\end{abstract}

\section{Introduction}

The "domination" was first used by Oystein Ore [1]. After that, studies on domination increased rapidly. The first textbook on dominance is published in 1998 [2]. There are lots of studies on domination but small number of them on edge-vertex domination.

Double edge-vertex domination is a quite new concept of domination. It was introduced in 2020. We studied on double edge-vertex domination of some basic graph classes, double edge-vertex domination of corona pruduct and double edge-vertex domination of cartesian product our previous studies [3, 4]. In this paper, we study on double edge-vertex domination of middle and splitting graphs of paths and cycle.

Let $G=(V, E)$ be a simple graph. The set $N(v)=\{v \in V \mid u v \in E\}$ is open neighborhood and $N[v]=N(v) \cup N(u)$ is closed neigboorhood of $v \in V$. For any edge $e \in E$, the open edge neighborhood $N(e)$ of $e$ is the set of edges adjacent to $e$ [5].

For $S \subseteq V$ of vertices in a graph $G=(V, E)$ is called a dominating set if every vertex $v \in V$ is either an element of $S$ or adjacent to an element of $S$. The minimum cardinality of a dominating set of $G$ is called the domination number and is denoted by $\gamma(G)$ [2].

A subset $X$ of $E$ is called an edge dominating set of $G$ if every edge not in $X$ is adjacent to some edge in $X$. The edge domination number $\gamma^{\prime}(G)$ of $G$ is the minimum cardinality taken over all edge dominating sets of $G$ [6].

A vertex $v$ of $G=(V, E)$ is said to be vertex-edge dominate every edge incident to $v$, as well as every edge adjacent to these incident edges. A set $S \subseteq V$ is a vertex-edge dominating set, if every edge of $E$ is vertex-edge dominated by at least one vertex of $S$. The minimum cardinality of a vertex-edge dominating set of $G$ is the vertex-edge domination number and is denoted by $\gamma_{v e}(G)[7]$.

An edge $e=u v$ of graph $G=(V, E)$ is said to be edge-vertex dominate vertices $u$ and $v$, as well as all vertices adjacent to $u$ and $v$. A set $S \subseteq E$ is an edge-vertex dominating set, if every vertex of $V$ is edge-vertex dominated by at least an edge of $S$. The minimum cardinality of an edge-vertex dominating set of $G$ is the edge-vertex domination number and is denoted by $\gamma_{e v}(G)$ $[8]$. 
A subset $D \subseteq V$ is a double vertex-edge dominating set of $G$ if every edge of $E$ is vertex-edge dominated by at least two vertices of $D$. The double vertex-edge domination number of $G$, is the minumum cardinality of a double vertex-edge dominating set of $G$ and is denoted by $\gamma_{d v e}(G)[9]$.

An edge $e=u v$ of graph $G=(V, E)$ is said to be edge-vertex dominate vertices $u$ and $v$, as well as all vertices adjacent to $u$ and $v$. A set $S \subseteq E$ is a double edge-vertex dominating set, if every vertex of $V$ is edge-vertex dominated by at least two edges of $S$. The minimum cardinality of a double edge-vertex dominating set of $G$ is the double edge-vertex domination number and is denoted by $\gamma_{d e v}(G)$ [3].

For each vertex $v$ of a graph $G$, take a new vertex $v^{\prime}$ and join $v^{\prime}$ to all vertices of $G$ adjacent to $v$. The graph $S(G)$ thus obtained is called the splitting graph of $G[10]$.

The middle graph of a graph $G$ is a graph whose vertex set is $V(G) \cup E(G)$, and two vertices are adjacent if they are adjacent edges of $\mathrm{G}$ or one is a vertex and other is an edge incident with it, and it is denoted by $M(G)[11]$.

\section{Double edge-vertex domination on middle graph of $P_{n}$ and $C_{n}$}

In this section, we study on double edge- domination on middle graphs of $P_{n}$ and $C_{n}$. We found results on double edge-vertex domination number of this graph class and we prove that.

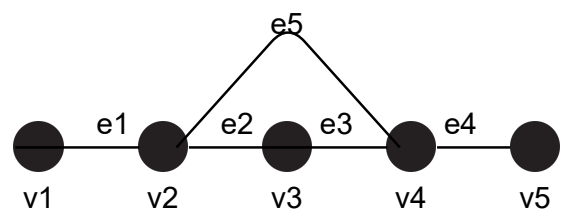

Figure 2.1: $M\left(P_{3}\right)$

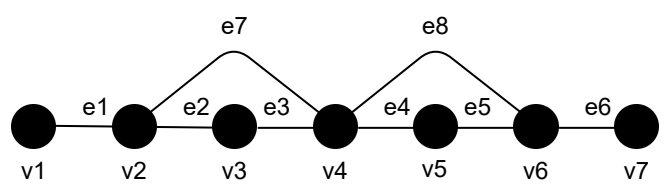

Figure 2.2: $M\left(P_{4}\right)$

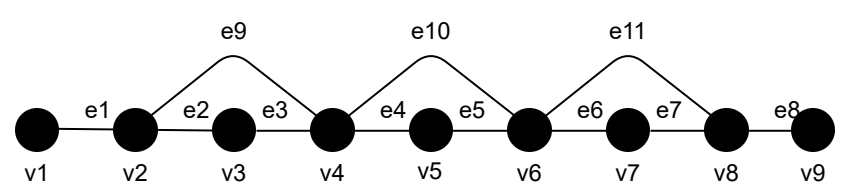

Figure 2.3: $M\left(P_{5}\right)$

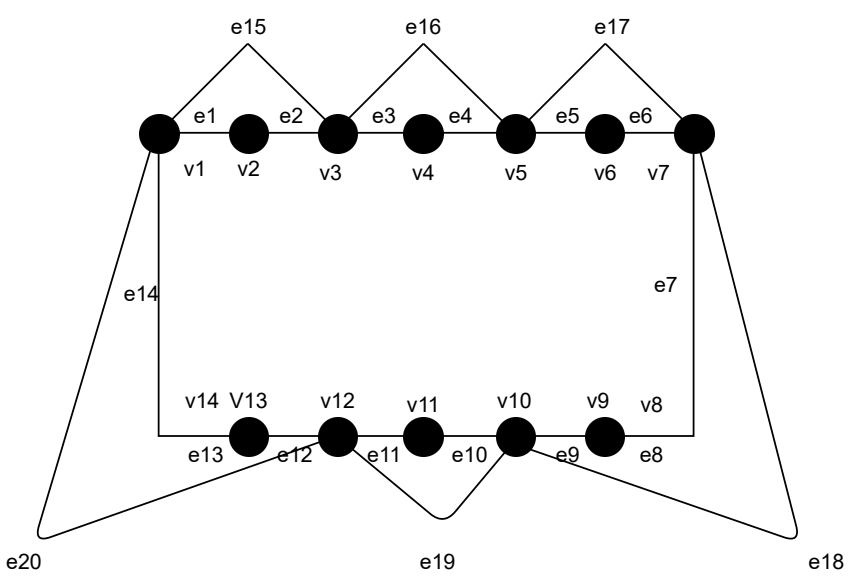

Figure 2.4: $M\left(C_{6}\right)$ 


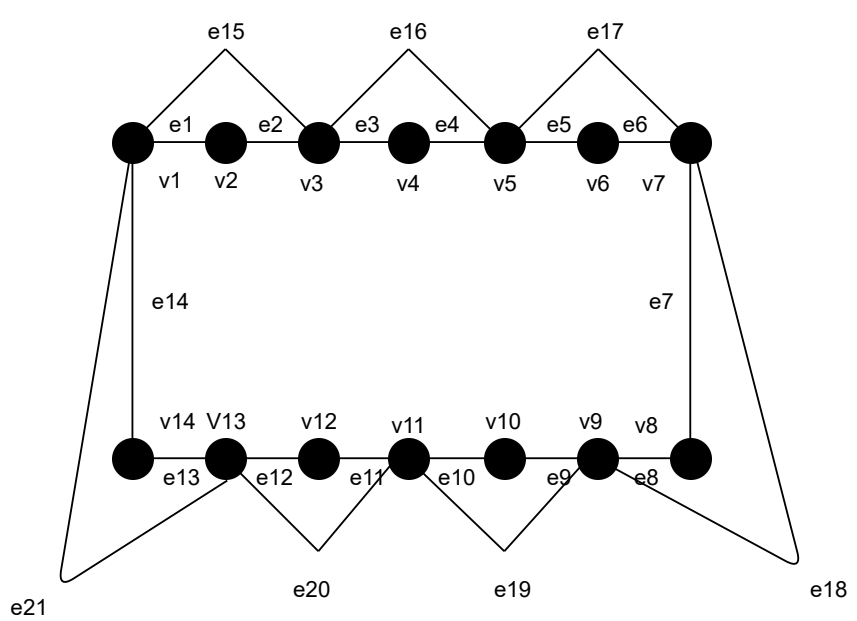

Figure 2.5: $M\left(C_{7}\right)$

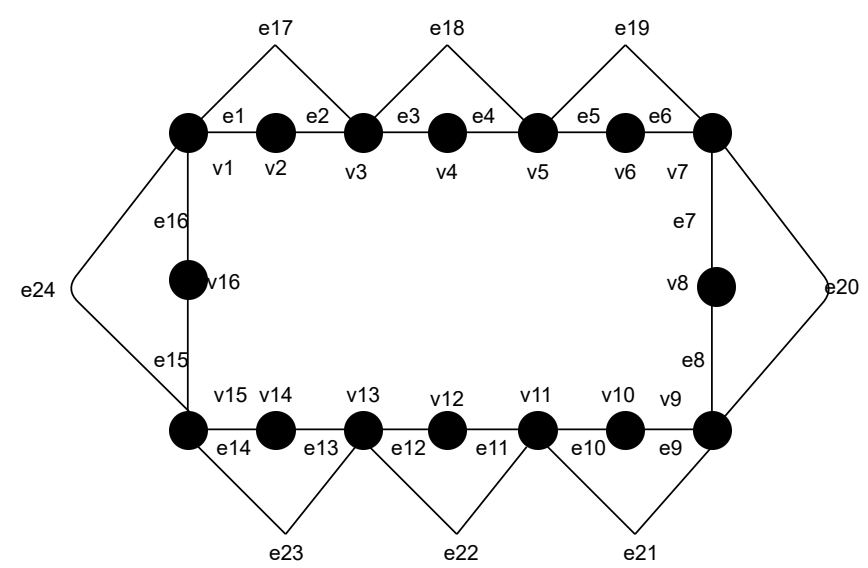

Figure 2.6: $M\left(C_{8}\right)$

Theorem 2.1. For a middle graph of path with order $n \geq 3$

$$
\gamma_{\text {dev }}\left(M\left(P_{n}\right)\right)=\left\{\begin{array}{c}
\frac{2 n}{3}+1, \text { if } n \equiv 0(\bmod 3) \\
\left\lceil\frac{2 n+2}{3}\right\rceil, \text { otherwise }
\end{array}\right.
$$

Proof. Let's prove this theorem by mathematical induction.

Case 1: For $n=3 k, n \equiv 0(\bmod 3)$, result is true for $n=3$.

$$
\gamma_{\text {dev }}\left(M\left(P_{3}\right)\right)=\frac{2.3}{3}+1=3
$$

We choose $e_{2}, e_{3}, e_{5}$ edges in Figure 2.1. Our assumption asserts that,

$$
\gamma_{d e v}\left(M\left(P_{3 k}\right)\right)=\frac{2.3 k}{3}+1
$$

Our goal is show that for $n=3(k+1)=3 k+3$, double edge-vertex domination number of middle graph of path is,

$$
\gamma_{d e v}\left(M\left(P_{3 k+3}\right)\right)=\frac{2 \cdot(3 k+3)}{3}+1
$$

We added 6 vertices to $3 k$, from Figure 2.1 we must choose at least two edges. Hence,

$$
\gamma_{d e v}\left(M\left(P_{3 k+3}\right)\right) \geq \gamma_{d e v}\left(M\left(P_{3 k}\right)+2\right.
$$




$$
=\frac{2.3 k}{3}+1+2=\frac{2.3 k}{3}+2+1=\frac{2.3 k}{3}+\frac{2}{1}+1=\frac{2.3 k}{3}+\frac{6}{3}+1=\frac{2.3 k+6}{3}+1=\frac{2.3 k+2.3}{3}+1=\frac{2 .(3 k+3)}{3}+1 .
$$

Case 2: For $n=3 k+1, n \equiv 1(\bmod 3)$, result is true for $n=4$.

$$
\gamma_{d e v}\left(M\left(P_{4}\right)\right)=\left\lceil\frac{2.4+2}{3}\right\rceil=4 .
$$

We choose $e_{2}, e_{5}, e_{7}, e_{8}$ edges in Figure 2.2. Our assumption asserts that,

$$
\gamma_{d e v}\left(M\left(P_{3 k+1}\right)\right)=\left\lceil\frac{2 \cdot(3 k+1)+2}{3}\right\rceil .
$$

We want to prove that for $n=3(k+1)+1=3 k+4$, double edge-vertex domination number of middle graph of path is,

$$
\gamma_{d e v}\left(M\left(P_{3 k+4}\right)\right)=\left\lceil\frac{2 \cdot(3 k+4)+2}{3}\right\rceil .
$$

We added 6 vertices to $3 k+1$, from Figure 2.2 we must choose at least two edges. Hence,

$$
\begin{gathered}
\gamma_{d e v}\left(M\left(P_{3 k+4}\right)\right) \geq \gamma_{d e v}\left(M\left(P_{3 k+1}\right)+2\right. \\
=\left\lceil\frac{2 \cdot(3 k+1)+2}{3}\right\rceil+2=\left\lceil\frac{2 \cdot(3 k+1)+2}{3}\right\rceil+\frac{6}{3} \geq\left\lceil\frac{2 \cdot(3 k+1)+2}{3}+\frac{6}{3}\right\rceil \\
=\left\lceil\frac{2 \cdot(3 k+1)+2+6}{3}\right\rceil=\left\lceil\frac{2 \cdot(3 k+1)+6+2}{3}\right\rceil=\left\lceil\frac{2 \cdot(3 k+1)+2 \cdot 3+2}{3}\right\rceil=\left\lceil\frac{2 \cdot(3 k+1+3)+2}{3}\right\rceil=\left\lceil\frac{2 \cdot(3 k+4)+2}{3}\right\rceil .
\end{gathered}
$$

Case 3: For $n=3 k+2, n \equiv 1(\bmod 3)$, result is true for $n=5$.

$$
\gamma_{d e v}\left(M\left(P_{5}\right)\right)=\left\lceil\frac{2.5+2}{3}\right\rceil=4
$$

We choose $e_{2}, e_{7}, e_{9}, e_{11}$ edges in Figure 2.3. Our assumption asserts that,

$$
\gamma_{d e v}\left(M\left(P_{3 k+2}\right)\right)=\left\lceil\frac{2 \cdot(3 k+2)+2}{3}\right\rceil .
$$

We want to prove that for $n=3(k+2)+1=3 k+5$, double edge-vertex domination number of middle graph of path is,

$$
\gamma_{d e v}\left(M\left(P_{3 k+5}\right)\right)=\left\lceil\frac{2 \cdot(3 k+5)+2}{3}\right\rceil .
$$

We added 6 vertices to $3 k+2$, from Figure 2.3 we must choose at least two edges. Hence,

$$
\begin{gathered}
\gamma_{d e v}\left(M\left(P_{3 k+5}\right)\right) \geq \gamma_{d e v}\left(M\left(P_{3 k+2}\right)+2\right. \\
=\left\lceil\frac{2 \cdot(3 k+2)+2}{3}\right\rceil+2=\left\lceil\frac{2 \cdot(3 k+2)+2}{3}\right\rceil+\frac{6}{3} \geq\left\lceil\frac{2 \cdot(3 k+2)+2}{3}+\frac{6}{3}\right\rceil \\
=\left\lceil\frac{2 \cdot(3 k+2)+2+6}{3}\right\rceil=\left\lceil\frac{2 \cdot(3 k+2)+6+2}{3}\right\rceil=\left\lceil\frac{2 \cdot(3 k+2)+2 \cdot 3+2}{3}\right\rceil=\left\lceil\frac{2 \cdot(3 k+2+3)+2}{3}\right\rceil=\left\lceil\frac{2 \cdot(3 k+5)+2}{3}\right\rceil .
\end{gathered}
$$


Theorem 2.2. For a middle graph of cycle with order $n \geq 6$

$$
\gamma_{\text {dev }}\left(M\left(C_{n}\right)\right)=\left\{\begin{array}{c}
\frac{2 n}{3}, \text { if } n \equiv 0(\bmod 3) \\
\left\lceil\frac{2 n+2}{3}\right\rceil-1, \text { otherwise }
\end{array}\right.
$$

Proof. Let's prove this theorem by mathematical induction.

Case 1: For $n=3 k, n \equiv 0(\bmod 3)$, result is true for $n=6$.

$$
\gamma_{d e v}\left(M\left(C_{6}\right)\right)=\frac{2.6}{3}=4
$$

We choose $e_{15}, e_{16}, e_{18}, e_{19}$ edges in Figure 2.4. Our assumption asserts that,

$$
\gamma_{d e v}\left(M\left(C_{3 k}\right)\right)=\frac{2.3 k}{3}
$$

We want to prove that for $n=3(k+1)=3 k+3$, double edge-vertex domination number of middle graph of cycle is,

$$
\gamma_{d e v}\left(M\left(C_{3 k+3}\right)\right)=\frac{2 \cdot(3 k+3)}{3}
$$

We added 6 vertices to $3 k$, from Figure 2.4 we must choose at least two edges. Hence,

$$
\begin{gathered}
\gamma_{d e v}(M(C 3 k+3)) \geq \gamma_{d e v}\left(M\left(C_{3 k}\right)=\frac{2 \cdot(3 k)}{3}+2=\frac{2 \cdot(3 k)}{3}+\frac{6}{3}\right. \\
=\frac{2 \cdot(3 k)+6}{3}=\frac{2 \cdot(3 k)+2 \cdot 3}{3}=\frac{2 \cdot(3 k+3)}{3} .
\end{gathered}
$$

Case 2: For $n=3 k+1, n \equiv 1(\bmod 3)$, result is true for $n=7$.

$$
\gamma_{d e v}\left(M\left(C_{7}\right)=\left\lceil\frac{2.7+2}{3}\right\rceil-1=\left\lceil\frac{14+2}{3}\right\rceil-1=\left\lceil\frac{16}{3}\right\rceil-1=6-1=5\right.
$$

We choose $e_{15}, e_{16}, e_{18}, e_{19}, e_{21}$ edges in Figure 2.5. Our assumption asserts that,

$$
\gamma_{d e v}\left(M\left(C_{3 k+1}\right)\right)=\left\lceil\frac{2 \cdot(3 k+1)+2}{3}\right\rceil-1 .
$$

We want to prove that for $n=3(k+1)+1=3 k+4$, double edge-vertex domination number of middle graph of cycle is,

$$
\gamma_{d e v}\left(M\left(C_{3 k+4}\right)\right)=\left\lceil\frac{2 .(3 k+4)+2}{3}\right\rceil-1 .
$$

We added 6 vertices to $3 k+1$, from Figure 2.5 we must choose at least two edges. Hence,

$$
\begin{gathered}
\gamma_{d e v}\left(M\left(C_{3 k+4}\right)\right) \geq \gamma_{d e v}\left(M\left(C_{3 k 12}\right)+2\right. \\
=\left\lceil\frac{2 \cdot(3 k+1)+2}{3}\right\rceil-1+2=\left\lceil\frac{2 \cdot(3 k+1)+2}{3}\right\rceil+2-1=\left\lceil\frac{2 \cdot(3 k+1)+2}{3}\right\rceil+\frac{6}{3} \geq\left\lceil\frac{2 \cdot(3 k+1)+2}{3}+\frac{6}{3}\right\rceil-1 \\
=\left\lceil\frac{2 \cdot(3 k+1)+2+6}{3}\right\rceil-1=\left\lceil\frac{2 \cdot(3 k+1)+6+2}{3}\right\rceil-1=\left\lceil\frac{2 \cdot(3 k+1)+2 \cdot 3+2}{3}\right\rceil-1=\left\lceil\frac{2 \cdot(3 k+1+3)+2}{3}\right\rceil-1 \\
=\left\lceil\frac{2 \cdot(3 k+4)+2}{3}\right\rceil-1 .
\end{gathered}
$$

Case 3: For $n=3 k+2, n \equiv 2(\bmod 3)$, result is true for $n=8$.

$$
\gamma_{d e v}\left(M\left(C_{8}\right)=\left\lceil\frac{2.8+2}{3}\right\rceil-1=\left\lceil\frac{16+2}{3}\right\rceil-1=\left\lceil\frac{18}{3}\right\rceil-1=6-1=5\right.
$$


We choose $e_{17}, e_{18}, e_{20}, e_{22}, e_{23}$ edges in Figure 2.6. Our assumption asserts that,

$$
\gamma_{d e v}\left(M\left(C_{3 k+2}\right)\right)=\left\lceil\frac{2 \cdot(3 k+2)+2}{3}\right\rceil-1 .
$$

We want to prove that for $n=3(k+2)+1=3 k+5$, double edge-vertex domination number of middle graph of cycle is,

$$
\gamma_{\text {dev }}\left(M\left(C_{3 k+5}\right)\right)=\left\lceil\frac{2 \cdot(3 k+5)+2}{3}\right\rceil-1 .
$$

We added 6 vertices to $3 k+2$, from Figure 2.6 we must choose at least two edges. Hence,

$$
\begin{gathered}
\gamma_{d e v}\left(M\left(C_{3 k+5}\right)\right) \geq \gamma_{d e v}\left(M\left(C_{3 k+2}\right)+2=\left\lceil\frac{2 \cdot(3 k+2)+2}{3}\right\rceil-1+2\right. \\
=\left\lceil\frac{2 \cdot(3 k+2)+2}{3}\right\rceil+2-1=\left\lceil\frac{2 \cdot(3 k+2)+2}{3}\right\rceil+\frac{6}{3} \geq\left\lceil\frac{2 \cdot(3 k+2)+2}{3}+\frac{6}{3}\right\rceil-1 \\
=\left\lceil\frac{2 .(3 k+2)+2+6}{3}\right\rceil-1=\left\lceil\frac{2 \cdot(3 k+2)+6+2}{3}\right\rceil-1=\left\lceil\frac{2 \cdot(3 k+2)+2 \cdot 3+2}{3}\right\rceil-1=\left\lceil\frac{2 \cdot(3 k+2+3)+2}{3}\right\rceil-1 \\
=\left\lceil\frac{2 \cdot(3 k+5)+2}{3}\right\rceil-1 .
\end{gathered}
$$

\section{Double edge-vertex domination on splitting graph of $P_{n}$ and $C_{n}$}

In this section, we study on splitting graph of $P_{n}$ and $C_{n}$. We found results on double edge-vertex domination number of this graph class and we prove that.

Theorem 3.1. For a splitting graph of path with order $n \geq 4$

$$
\gamma_{\text {dev }}\left(S\left(P_{n}\right)\right)=\left\{\begin{array}{l}
\left\lceil\frac{2 n}{4}\right\rceil, \text { if } n \equiv 3(\bmod 4) \\
\left\lceil\frac{2 n}{4}\right\rceil+1, \text { otherwise }
\end{array}\right.
$$

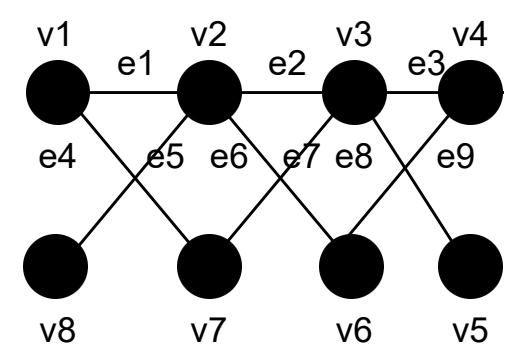

Figure 3.1: $S\left(P_{4}\right)$ 


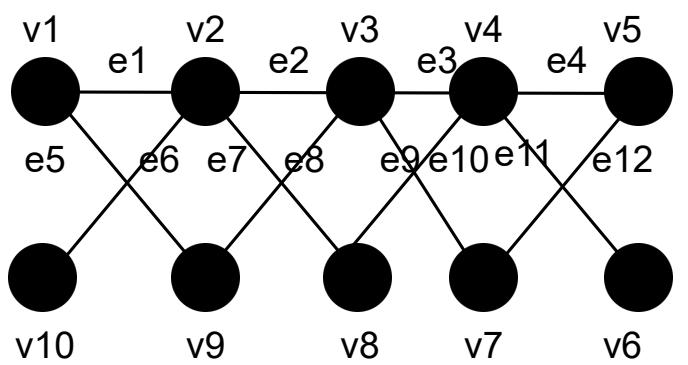

Figure 3.2: $S\left(P_{5}\right)$

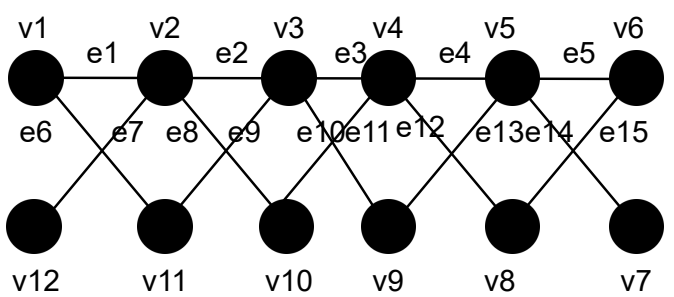

Figure 3.3: $S\left(P_{6}\right)$

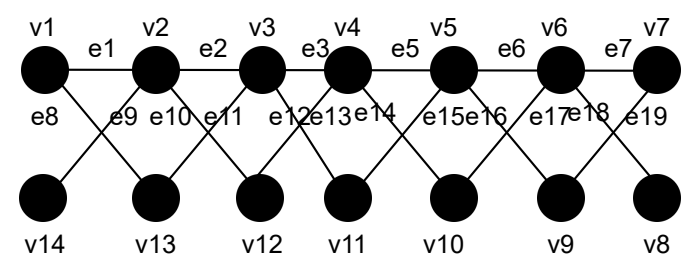

Figure 3.4: $S\left(P_{7}\right)$

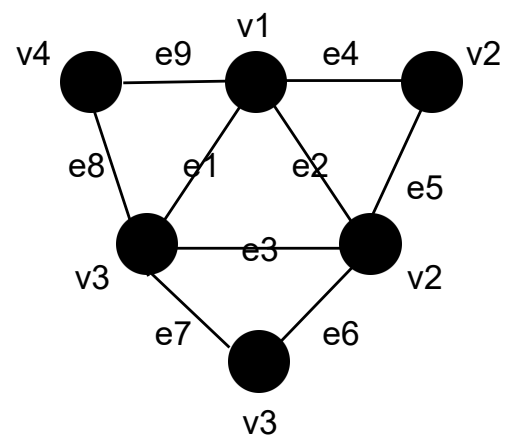

Figure 3.5: $S\left(C_{3}\right)$

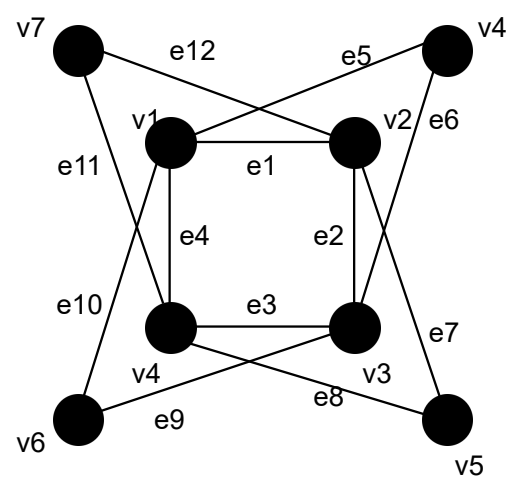

Figure 3.6: $S\left(C_{4}\right)$ 
Proof. We begin the selection edges of $P_{n}$, because degree of the edge of $P_{n}$ are the most.

Case 1: For $n=4 k, n \equiv 0(\bmod 4)$, result is true for $n=4$.

$$
\gamma_{\text {dev }}\left(S\left(P_{4}\right)\right)=\left\lceil\frac{2.4}{4}\right\rceil+1=3 .
$$

We choose $e_{1}, e_{2}, e_{3}$ edges in Figure 3.1. Our assumption asserts that,

$$
\gamma_{d e v}\left(S\left(P_{4 k}\right)\right)=\left\lceil\frac{2.4 k}{4}\right\rceil+1 .
$$

We want tho prove that for $n=4(k+1)=4 k+4$, double edge-vertex domination number of splitting graph of path is,

$$
\gamma_{d e v}\left(S\left(P_{4 k+4}\right)\right)=\left\lceil\frac{2 .(4 k+4)}{4}\right\rceil+1 .
$$

We added 8 vertices to $4 k$, from Figure 3.1 we must choose at least two edges. Hence,

$$
\begin{gathered}
\gamma_{d e v}\left(S\left(P_{4 k+4}\right)\right) \geq \gamma_{d e v}\left(S\left(P_{4 k}\right)+2\right. \\
=\left\lceil\frac{2.4 k}{4}\right\rceil+1+2=\left\lceil\frac{2.4 k}{4}\right\rceil+2+1=\left\lceil\frac{2.4 k}{4}\right\rceil+\frac{8}{4}+1 \geq\left\lceil\frac{2.4 k}{4}+\frac{8}{4}\right\rceil+1 \\
=\left\lceil\frac{2.4 k+2.4}{4}\right\rceil+1=\left\lceil\frac{2.4 k+4}{4}\right\rceil+1 .
\end{gathered}
$$

Case 2: For $n=4 k+1, n \equiv 1(\bmod 4)$, result is true for $n=5$.

$$
\gamma_{\text {dev }}\left(S\left(P_{5}\right)\right)=\left\lceil\frac{2.5}{4}\right\rceil+1=3+1=4
$$

We choose $e_{1}, e_{2}, e_{3}, e_{4}$ edges in Figure 3.2. Our assumption asserts that,

$$
\gamma_{d e v}\left(S\left(P_{4 k+1}\right)\right)=\left\lceil\frac{2 .(4 k+1)}{4}\right\rceil+1 .
$$

We want the prove that for $n=4(k+1)+1=4 k+5$, double edge-vertex domination number of splitting graph of path is,

$$
\gamma_{d e v}\left(S\left(P_{4 k+5}\right)\right)=\left\lceil\frac{2 .(4 k+5)}{4}\right\rceil+1
$$

We added 8 vertices to $4 k+1$, from Figure 3.2 we must choose at least two edges. Hence,

$$
\begin{gathered}
\gamma_{\text {dev }}\left(S\left(P_{4 k+5}\right)\right) \geq \gamma_{\text {dev }}\left(S\left(P_{4 k+1}\right)+2\right. \\
=\left\lceil\frac{2 \cdot(4 k+1)}{4}\right\rceil+1+2=\left\lceil\frac{2 \cdot(4 k+1)}{4}\right\rceil+2+1=\left\lceil\frac{2 \cdot(4 k+1)}{4}\right\rceil+\frac{8}{4}+1 \geq\left\lceil\frac{2 \cdot(4 k+1)}{4}+\frac{8}{4}\right\rceil+1 \\
=\left\lceil\frac{2 \cdot(4 k+1)+8}{4}\right\rceil+1=\left\lceil\frac{2 \cdot(4 k+1)+2 \cdot 4}{4}\right\rceil+1=\left\lceil\frac{2 \cdot(4 k+1+4)}{4}\right\rceil+1=\left\lceil\frac{2 \cdot(4 k+5)}{4}\right\rceil+1 .
\end{gathered}
$$

Case 3: For $n=4 k+2, n \equiv 2(\bmod 4)$, result is true for $n=6$.

$$
\gamma_{\text {dev }}\left(S\left(P_{6}\right)\right)=\left\lceil\frac{2.6}{4}\right\rceil+1=3+1=4 .
$$

We choose $e_{1}, e_{2}, e_{3}, e_{4}, e_{5}$ edges in Figure 3.3. Our assumption asserts that,

$$
\gamma_{d e v}\left(S\left(P_{4 k+2}\right)\right)=\left\lceil\frac{2 .(4 k+2)}{4}\right\rceil+1 .
$$


We want the prove that for $n=4(k+2)+1=4 k+6$, double edge-vertex domination number of splitting graph of path is,

$$
\gamma_{d e v}\left(S\left(P_{4 k+6}\right)\right)=\left\lceil\frac{2 .(4 k+6)}{4}\right\rceil+1 .
$$

We added 8 vertices to $4 k+2$, from Figure 3.3 we must choose at least two edges. Hence,

$$
\begin{gathered}
\gamma_{\text {dev }}\left(S\left(P_{4 k+6}\right)\right) \geq \gamma_{\text {dev }}\left(S\left(P_{4 k+2}\right)+2\right. \\
=\left\lceil\frac{2 \cdot(4 k+2)}{4}\right\rceil+1+2=\left\lceil\frac{2 \cdot(4 k+2)}{4}\right\rceil+2+1=\left\lceil\frac{2 \cdot(4 k+2)}{4}\right\rceil+\frac{8}{4}+1 \geq\left\lceil\frac{2 \cdot(4 k+2)}{4}+\frac{8}{4}\right\rceil+1 \\
=\left\lceil\frac{2 \cdot(4 k+2)+8}{4}\right\rceil+1=\left\lceil\frac{2 \cdot(4 k+2)+2 \cdot 4}{4}\right\rceil+1=\left\lceil\frac{2 \cdot(4 k+2+4)}{4}\right\rceil+1=\left\lceil\frac{2 \cdot(4 k+6)}{4}\right\rceil+1 .
\end{gathered}
$$

Case 4: For $n=4 k+3, n \equiv 3(\bmod 4)$, result is true for $n=7$.

$$
\gamma_{\text {dev }}\left(S\left(P_{7}\right)\right)=\left\lceil\frac{2.7}{4}\right\rceil=4
$$

We choose $e_{1}, e_{2}, e_{3}, e_{5}, e_{6}, e_{7}$ edges in Figure 3.4. Our assumption asserts that,

$$
\gamma_{d e v}\left(S\left(P_{4 k+3}\right)\right)=\left\lceil\frac{2 .(4 k+3)}{4}\right\rceil .
$$

We want the prove that for $n=4(k+3)+1=4 k+7$, double edge-vertex domination number of splitting graph of path is,

$$
\gamma_{\text {dev }}\left(S\left(P_{4 k+7}\right)\right)=\left\lceil\frac{2 .(4 k+7)}{4}\right\rceil .
$$

We added 8 vertices to $4 k+3$, from (3.1) we must choose at least two edges. Hence,

$$
\begin{gathered}
\gamma_{d e v}\left(S\left(P_{4 k+7}\right)\right) \geq \gamma_{d e v}\left(S\left(P_{4 k+3}\right)+2\right. \\
=\left\lceil\frac{2 .(4 k+3)}{4}\right\rceil+2=\left\lceil\frac{2 \cdot(4 k+3)}{4}\right\rceil+2=\left\lceil\frac{2 \cdot(4 k+3)}{4}\right\rceil+\frac{8}{4} \geq\left\lceil\frac{2 \cdot(4 k+3)}{4}+\frac{8}{4}\right\rceil \\
=\left\lceil\frac{2 .(4 k+3)+8}{4}\right\rceil=\left\lceil\frac{2 \cdot(4 k+3)+2.4}{4}\right\rceil=\left\lceil\frac{2 \cdot(4 k+3+4)}{4}\right\rceil=\left\lceil\frac{2 \cdot(4 k+7)}{4}\right\rceil .
\end{gathered}
$$

Theorem 3.2. For a splitting graph of cycle with order $n \geq 3$

$$
\gamma_{\text {dev }}\left(S\left(C_{n}\right)\right)=\left\lceil\frac{n}{2}\right\rceil \text {. }
$$

Proof. We begin the selection edges of $C_{n}$, because degree of the edge of $C_{n}$ are the most.

Case 1: For $n=2 k+1$, result is true for $n=3$.

$$
\gamma_{d e v}\left(S\left(C_{3}\right)\right)=\left\lceil\frac{3}{2}\right\rceil=2 .
$$

We choose $e_{1}, e_{2}$ edges in Figure 3.5. Our assumption asserts that,

$$
\gamma_{d e v}\left(S\left(C_{2 k+1}\right)\right)=\left\lceil\frac{2 k+1}{2}\right\rceil .
$$

We want the prove that for $n=2(k+1)+1=2 k+3$, double edge-vertex domination number of splitting graph of cycle is,

$$
\gamma_{d e v}\left(M\left(C_{2 k+3}\right)\right)=\left\lceil\frac{2 k+3}{2}\right\rceil .
$$


We added 2 vertices to $2 k+1$, therefore we must choose at least one edge. Hence,

$$
\begin{gathered}
\gamma_{d e v}\left(M\left(C_{2 k+3}\right)\right) \geq \gamma_{d e v}\left(M\left(C_{2 k+1}\right)+1\right. \\
=\left\lceil\frac{2 k+1}{2}\right\rceil+1=\left\lceil\frac{2 k+1}{2}\right\rceil+\frac{2}{2} \geq\left\lceil\frac{2 k+1}{2}+\frac{2}{2}\right\rceil=\left\lceil\frac{2 k+1+2}{2}\right\rceil \\
=\left\lceil\frac{2 k+3}{2}\right\rceil .
\end{gathered}
$$

Case 2: For $n=2 k$, result is true for $n=4$.

$$
\gamma_{\text {dev }}\left(M\left(C_{4}\right)\right)=\left\lceil\frac{4}{2}\right\rceil=2 .
$$

We choose $e_{1}, e_{3}$ edges in Figure 3.6. Our assumption asserts that,

$$
\gamma_{d e v}\left(M\left(C_{2 k}\right)\right)=\left\lceil\frac{2 k}{2}\right\rceil .
$$

We want the prove that for $n=2(k+1)=2 k+2$, double edge-vertex domination number of splitting graph of cycle is,

$$
\gamma_{d e v}\left(M\left(C_{2 k+2}\right)\right)=\left\lceil\frac{2 k+2}{2}\right\rceil
$$

We added 2 vertices to $2 k$, therefore we must choose at least one edge. Hence,

$$
\begin{gathered}
\gamma_{d e v}\left(M\left(C_{2 k+2}\right)\right) \geq \gamma_{d e v}\left(M\left(C_{2 k}\right)+1\right. \\
=\left\lceil\frac{2 k}{2}\right\rceil+1=\left\lceil\frac{2 k}{2}\right\rceil+\frac{2}{2}=\left\lceil\frac{2 k}{2}+\frac{2}{2}\right\rceil=\left\lceil\frac{2 k+2}{2}\right\rceil .
\end{gathered}
$$

\section{Conclusion}

Graphs, can be used to model communication networks. We can represent any network with graphs. Each center in the structure of the network is represented as a vertex, and each connection as an edge in graph. For axample, a telecommunication system, GPS/Google maps, connections of social media and many more can be represented as a graph. It is important to ensure persistence of communication in networks. In graph theory, various metrics are used to strengthen the stability of communication networks. Domination is one of the most studied topics of them. Domination has wide range of applications. For instance, to compute a wireless network with minimal power, to decide the locations where the radio stations in the region will be located so that the radio messages can be broadcast to all places in the region, to define the route of shuttle buses. There are lots of studies on domination concept. We have also worked on topic in our previous studies of double edge-vertex domination. In this study we find double edge-vertex domination number of middle and splitting graph of $P_{n}$ and $C_{n}$. In future study we plan to generalize the results on middle and splitting graphs of any given graphs.

\section{Acknowledgements}

The authors would like to express their sincere thanks to the editor and the anonymous reviewers for their helpful comments and suggestions.

\section{Funding}

There is no funding for this work.

\section{Availability of data and materials}

Not applicable.

\section{Competing interests}

The authors declare that they have no competing interests. 


\section{Author's contributions}

All authors contributed equally to the writing of this paper. All authors read and approved the final manuscript.

\section{References}

[1] O. Ore, Theory of graphs, American Mathematical Society, Providence, RI, 30 (1962), 206-212.

[2] T.W. Haynes, S.T. Hedetniemi, P.J. Slater, Fundamentals of Domination in Graphs, Marcel Dekker Inc., New York, 1998.

[3] E. Kılıç, B. Ayl1, Double edge-vertex domination number of graphs, Adv. Math. Mod. Appl., 5(1), (2020), 19-37.

[4] E. Kilıç, B. Ayl1, Double edge-vertex domination under some graph operations, J. Mod. Tech. Eng., 5(2), (2020), 175-180

[5] V.R. Kulli, The neighborhood graph of a graph, International Journal of Fuzzy Mathematical Archive, 8(2), (2015), 93-99.

[6] S. Mitchell, S.T. Hedetniemi, Edge domination in trees, Congr. Numer., 19, (1977), 489-509.

[7] J.W. Peters, Theoretical and algorithmic results on domination and connectivity, Ph.D.Thesis, Clemson University, 1986.

[8] J.R. Lewis, Edge and edge-vertex domination in graphs, Ph.D. Thesis, Clemson University, 2007.

[9] B. Krishnakumari, M. Chellali, Y.B. Venkatakrishnan, Double vertex-edge domination, Disc. Math., Algorithms and Applications, 9(4), (2017), Article ID 1750045 , doi:10.1142/S1793830917500458.

[10] E. Sampathkumar, H.B. Walikar, On splitting graph of a graph, J. Karnatak Univ. Sci., 25(13), (1980), 13-16.

[11] I. Hamada, T. Yoshimura, Traversability and connectivity of the middle graph of a graph, Disc. Math., 14(3), (1976), 247-256. 